7 Gass JDM, Slamovits TL, Fuller DG, Gieser RG, Lean JS Posterior chorioretinopathy and retinal detachment after organ transplantation. Arch Ophthalmol 1992; 110: 1717-1722.

8 Gass JDM, Little H. Bilateral bullous exudative retinal detachments complicating idiopathic central serous chorioretinopathy during systemic corticosteroid therapy. Ophthalmology 1995; 102: 737-747.

AD Singh, H Demirci, CL Shields and JA Shields

\author{
Oncology Service \\ Wills Eye Hospital \\ Thomas Jefferson University \\ Philadelphia, PA, USA
}

Correspondence: AD Singh

Royal Hallamshire Hospital

Glossop Road, Sheffield S10 2JF, UK

Tel: +1142712902

Fax: 1142766381

E-mail: arunsingh@eyetumors.com

Sir,

\section{Multifocal asymptomatic retinal pigment epithelial detachments in neurosyphilis}

Eye (2003) 17, 524-525. doi:10.1038/sj.eye.6700381

Syphilis is a great imitator with protean ocular and systemic manifestations. It has been associated with a variety of posterior segment lesions. Reported manifestations include chorioretinitis, vasculitis, choroidal neovascular membrane, pigmentary retinopathy, retinal necrosis, retinal detachment, uveal effusion, central retinal vein occlusion, and big blind spot syndrome. ${ }^{1}$ We report here a patient of neurosyphilis who presented with multifocal asymptomatic retinal pigment epithelium (RPE) detachments. To the best of our knowledge, there is no previous report of RPE detachment or the related finding ${ }^{2}$ of central serous chorioretinopathy (CSC) in syphilis.

\section{Case report}

A 41-year-old male presented to the accident and emergency unit with generalized seizures. A diagnosis of neurosyphilis was made on the basis of positive serum and CSF VDRL along with positive TPHA and FTA-ABS. HIV testing was negative. He responded to a 17-day course of procaine penicillin with oral probenecid. Systemic steroids were not used in his treatment at any time. He was routinely referred to ophthalmology to rule out ocular involvement.

He had no ocular symptoms. Unaided visual acuities were $6 / 5$. There was no evidence of present or past intraocular inflammation. Fundus examination revealed bilateral, multifocal, well-circumscribed retinal pigment epithelium detachments. There were multiple lesions in both eyes. The right eye had one large detachment, about six disc diameters in size along the inferotemporal arcade (Figure 1). There were two smaller detachments, about a half disc diameter in size nasal to the fovea (Figure 1), and another about one disc diameter in size nasal to the disc. The left eye also had a large lesion, about four disc diameters in size, along the inferotemporal arcade (Figure 2) and another smaller one along the superotemporal arcade. There were bilateral scattered areas of pigment mottling and hypopigmentation. Fluorescein angiography showed early homogeneous filling of the detachments (Figure 2, top), with increase in intensity but not size of hyperfluorescence in the late phase (Figure 2, bottom), confirming the diagnosis of RPE detachment. Some scattered areas of localized hyperfluorescence were also present in both eyes, consistent with RPE window defects (Figure 2).

The patient was followed up at regular intervals. The detachments gradually subsided. At the last follow-up, 2 years after presentation, all the lesions had resolved. The patient maintained his vision at $6 / 5$ bilaterally, with no evidence of inflammation in either eye.

\section{Comment}

Idiopathic serous detachments of the RPE in patients below 50 years of age are thought to be variants of CSC. ${ }^{2}$ Although the pathophysiology remains controversial, most agree that increased choroidal vascular permeability is a prerequisite. ${ }^{2,3}$ It has further been suggested that choroidal ischaemia may be the primary event responsible for the altered permeability. ${ }^{4}$ This model of CSC suggests that ischaemia at the level of the

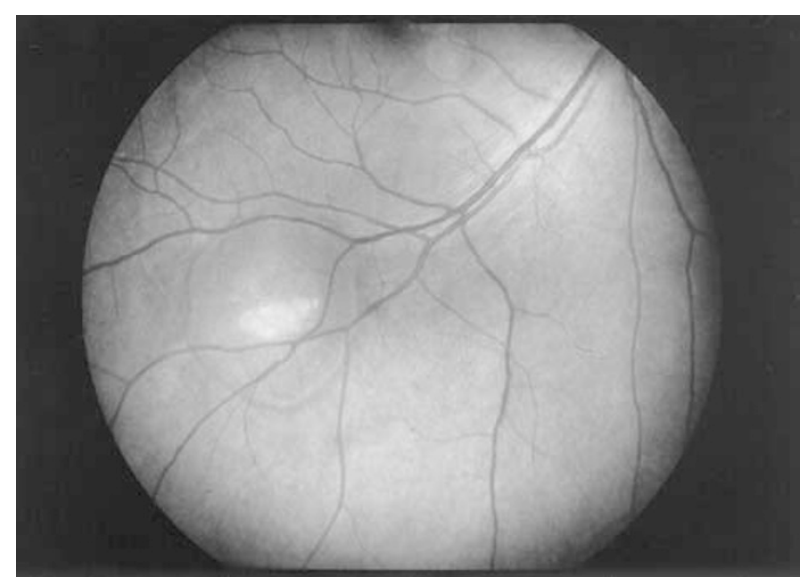

Figure 1 RPE detachments along the inferotemporal arcade and nasal to the macula, right eye. 

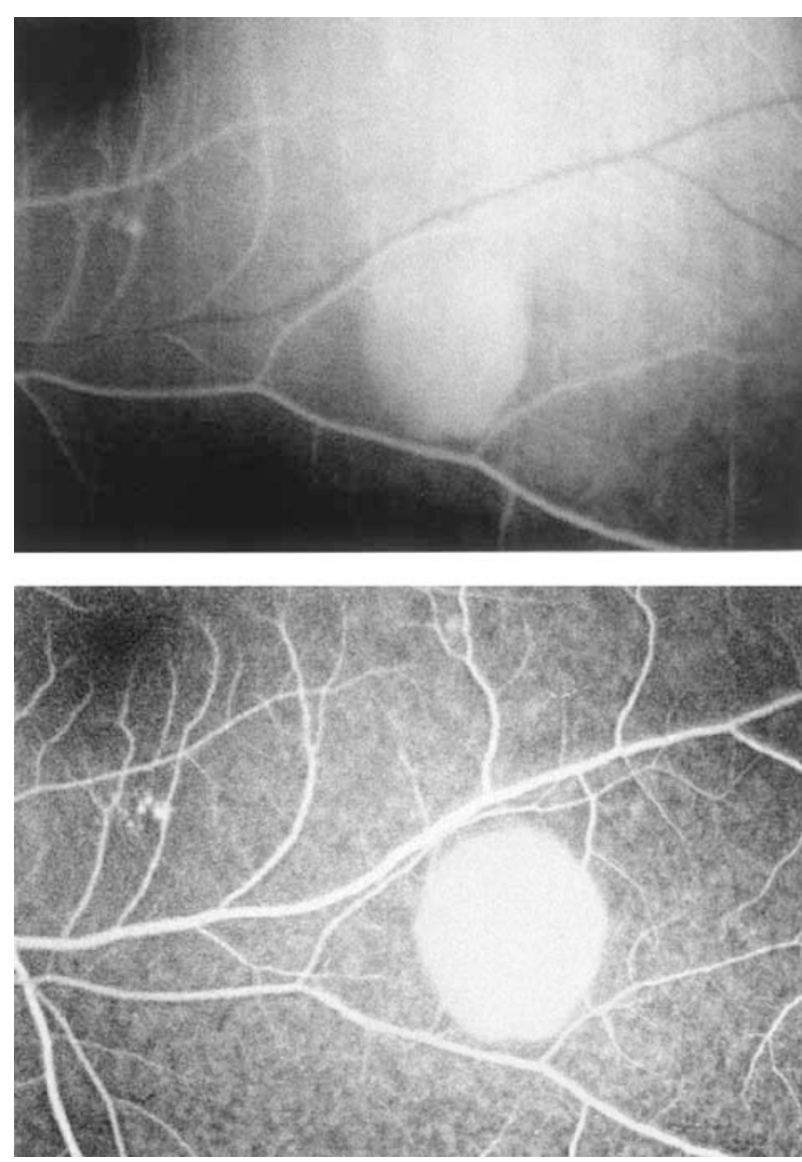

Figure 2 Fluorescein angiogram, left eye, showing (top) early homogeneous filling of pigment epithelial detachment and (bottom) increase in intensity but not size of hyperfluorescence in the late phase. Also seen are areas of RPE window defects inferior to the fovea.

choroid may either result in localized RPE changes such as pigment mottling and clumping or cause capillary and venous congestion with increased fluid transudation and RPE detachment. ${ }^{4}$ Both types of lesions were present in our patient.

The histological hallmark of syphilis is obliterative endarteritis affecting the small arteries and arterioles. ${ }^{5}$ At the level of choroidal vasculature, this would lead to choroidal ischaemia and might explain the ocular findings seen in our patient. However, ocular manifestations of syphilis are known to resolve rapidly with systemic treatment. Pigment epithelial detachments on the other hand, by their nature, would take time to settle once formed. This would explain the slow resolution of ocular findings in this case. The fact that no new detachments appeared over a 2-year follow-up supports the presence of an initial triggering event.

Unfortunately, there is no non-invasive method of establishing aetiology and the coincidental co-occurrence of these two events cannot be ruled out. Much more clinical evidence would be needed if a causal relationship was to be established.

In conclusion, we report here an interesting finding of multifocal asymptomatic RPE detachments in a patient of neurosyphilis.

\section{Acknowledgement}

Neither author has proprietary interest in the manuscript.

\section{References}

1 Margo CE, Hamed CM. Ocular syphilis. Surv Ophthalmol 1992; 37: 203-204.

2 Giovanni A, Scassellati-Sforzolini B, D'Altobrando E et al. Choroidal findings in the course of idiopathic serous pigment epithelium detachment detected by indocyanine green videoangiography. Retina 1997; 17: 286-293.

3 Uyama M, Matsunaga H, Matsubara T et al. Indocyanine green angiography and pathophysiology of multifocal posterior pigment epitheliopathy. Retina 1999; 19: 12-21.

4 Prunte C, Flammer J. Choroidal capillary and venous congestion in central serous chorioretinopathy. Am J Ophthalmol 1996; 12: 26-34.

5 von Lichtenberg F. Infectious disease. In: Cotran R, Kumar M, Robbins SL (eds). Robbins Pathologic Basis of Disease. WB Saunders Company: Philadelphia, 1989, pp. 368-371.

\section{S Anand and AS Mushin}

Department of Ophthalmology

Royal London Hospital

Whitechapel

London E1 1BB, UK

\section{Correspondence: Dr S Anand}

\section{3, Croft Gardens}

Birkby

Huddersfield

HD2 2FL

Tel: +447976907456

E-mail: seema180@hotmail.com

Sir,

Spontaneous suprachoroidal haemorrhage associated with high myopia and aspirin

Eye (2003) 17, 525-527. doi:10.1038/sj.eye.6700388

Spontaneous suprachoroidal haemorrhage occurs in age-related macular degeneration (ARMD) and anticoagulants are a recognised risk factor. ${ }^{1}$ In highly 